PROCEEDINGS OF THE

AMERICAN MATHEMATICAL SOCIETY

Volume 131, Number 1, Pages 35-40

S 0002-9939(02)06506-1

Article electronically published on May 13, 2002

\title{
A BLOCK THEORETIC ANALOGUE OF A THEOREM OF GLAUBERMAN AND THOMPSON
}

\author{
RADHA KESSAR AND MARKUS LINCKELMANN
}

(Communicated by Stephen D. Smith)

\begin{abstract}
If $p$ is an odd prime, $G$ a finite group and $P$ a Sylow- $p$-subgroup of $G$, a theorem of Glauberman and Thompson states that $G$ is $p$-nilpotent if and only if $N_{G}(Z(J(P)))$ is $p$-nilpotent, where $J(P)$ is the Thompson subgroup of $P$ generated by all abelian subgroups of $P$ of maximal order. Following a suggestion of G. R. Robinson, we prove a block-theoretic analogue of this theorem.
\end{abstract}

Theorem. Let $p$ be an odd prime and let $k$ be an algebraically closed field of characteristic $p$. Let $G$ be a finite group, $b$ a block of $k G$, and $P$ a defect group of $b$. Set $N=N_{G}(Z(J(P)))$ and let $c$ be the unique block of $k N$ such that $\operatorname{Br}_{P}(c)=\operatorname{Br}_{P}(b)$; that is, $c$ is the Brauer correspondent of $b$. Then $k G b$ is nilpotent if and only if $k N c$ is nilpotent.

We refer to [5] and [7] for accounts on the terminology from group theory and block theory, respectively, involved in the theorem above and its proof. Nilpotent blocks, introduced by Broue and Puig in [3], are the block theoretic analogue of the notion of $p$-nilpotent groups; the principal block of $k G$ is nilpotent if and only if $G$ is $p$-nilpotent. Thus, in this case, our theorem is equivalent to the theorem of Glauberman and Thompson. The proof proceeds in two steps. We reduce to the case where $G$ is the normaliser of a $b$-centric Brauer pair (following the lines of the proof of [5. Ch. 8, Theorem 3.1]), and then we apply results of Külshammer and Puig in [6] to transport the problem back to the analogous group theoretic statement.

Proof. We fix a block $e_{P}$ of $k C_{G}(P)$ such that $\operatorname{Br}_{P}(b) e_{P}=e_{P}$; that is, $\left(P, e_{P}\right)$ is a maximal $b$-Brauer pair. By [1], for any subgroup $Q$ of $P$ there is a unique block $e_{Q}$ of $k C_{G}(Q)$ such that $\left(Q, e_{Q}\right) \subseteq\left(P, e_{P}\right)$. Denote by $\mathcal{F}_{G, b}$ the category whose objects are the subgroups of $P$ and whose set of morphisms from a subgroup $Q$ of $P$ to another subgroup $R$ of $P$ is the set of group homomorphisms $\varphi: Q \rightarrow R$ for which there exists an element $x \in G$ satisfying $\varphi(u)=x u x^{-1}$ for all $u \in Q$ and ${ }^{x}\left(Q, e_{Q}\right) \subseteq\left(R, e_{R}\right)$. Thus the automorphism group of a subgroup $Q$ of $P$ as an object of the category $\mathcal{F}_{G, b}$ is canonically isomorphic to $N_{G}\left(Q, e_{Q}\right) / C_{G}(Q)$. By Alperin's fusion theorem, the category $\mathcal{F}_{G, b}$ is completely determined by the structure of $P$ and the groups $N_{G}\left(Q, e_{Q}\right) / C_{G}(Q)$ where either $Q=P$ or $\left(Q, e_{Q}\right)$ is

Received by the editors June 14, 2001 and, in revised form, August 15, 2001.

2000 Mathematics Subject Classification. Primary 20C20.

(C)2002 American Mathematical Society 
an essential $b$-Brauer pair (cf. 7, $\S 48]$ ). Note that $O_{p}(G) \subseteq Q$ whenever the pair $\left(Q, e_{Q}\right)$ is essential.

By Brauer's third main theorem (cf. [7, (40.17)]), if $b$ is the principal block of $k G$, then $e_{Q}$ is the principal block of $k C_{G}(Q)$, for any subgroup $Q$ of $P$. Thus the above condition ${ }^{x}\left(Q, e_{Q}\right) \subseteq\left(R, e_{R}\right)$ is equivalent to ${ }^{x} Q \subseteq R$. Therefore, if $b$ is the principal block of $k G$, we write $\mathcal{F}_{G}$ instead of $\mathcal{F}_{G, b}$.

In general, the definition of $\mathcal{F}_{G, b}$ depends on the choice of a maximal $b$-Brauer pair, but since all maximal $b$-Brauer pairs are $G$-conjugate, it is easy to see that $\mathcal{F}_{G, b}$ is unique up to isomorphism of categories. Note that we always have $\mathcal{F}_{P} \subseteq \mathcal{F}_{G, b}$. Following [3], the block $b$ is called nilpotent if $\mathcal{F}_{P}=\mathcal{F}_{G, b}$.

If $H$ is any subgroup of $G$ containing $P C_{G}(P)$, the block $e_{P}$ determines a unique block $d$ of $k H$ by $\operatorname{Br}_{P}(d) e_{P}=e_{P}$. Then $\left(P, e_{P}\right)$ is also a maximal $d$-Brauer pair, and this gives rise to the Brauer category $\mathcal{F}_{H, d}$ of $k H d$, defined as above for $H$ and $d$ instead of $G$ and $b$.

We are going to frequently use the following fact:

1. If $Q$ is a normal subgroup of $P$ and $H$ a subgroup of $G$ such that $P C_{G}(Q) \subseteq$ $H \subseteq N_{G}(Q)$, then

$$
\mathcal{F}_{H, d} \subseteq \mathcal{F}_{G, b},
$$

where $d$ is the unique block of $k H$ such that $\operatorname{Br}_{P}(d) e_{P}=e_{P}$. In particular, if $k G b$ is nilpotent, then $k H d$ is nilpotent.

Proof. If $\left(R, f_{R}\right)$ is an essential $d$-Brauer pair contained in $\left(P, e_{P}\right)$, then $R$ contains $Q$ as $Q$ is normal in $H$. But then $C_{G}(R)=C_{H}(R)$, and hence $f_{R}=e_{R}$. Thus $N_{H}\left(R, f_{R}\right) / C_{H}(R)$ is a subgroup of $N_{G}\left(R, e_{R}\right) / C_{G}(R)$.

Statement 1 applies to $N, c$ and $Z(J(P))$ instead of $H, d, Q$, respectively. Thus if $k G b$ is nilpotent, so is $k N c$. In order to show the converse, we consider now a minimal counterexample; that is, we assume that $k G b$ is not nilpotent while $k N c$ is nilpotent and that $|G|$ is minimal with this property. Under this assumption, 1 implies the following statement:

2. If $Q$ is a normal subgroup of $P$ and $H$ a subgroup of $G$ such that $P C_{G}(Q) \subseteq$ $H \subseteq N_{G}(Q)$, then either $H=G$ or $k H d$ is nilpotent, where $d$ is the unique block of $k H$ such that $\operatorname{Br}_{P}(d) e_{P}=e_{P}$.

Proof. Let $e$ be the unique block of $N \cap H$ such that $\operatorname{Br}_{P}(e) e_{P}=e_{P}$. We have $P C_{N}(Q) \subseteq N \cap H \subseteq N_{N}(Q)$, and thus statement 1 implies that $\mathcal{F}_{N \cap H, e} \subseteq \mathcal{F}_{N, c}$. But then $k(N \cap H) e$ is nilpotent, as $k N c$ is also. Therefore, if $H$ is a proper subgroup of $G$, then the induction hypothesis implies that the block $k H d$ is nilpotent.

3. We have $O_{p}(G) \neq\{1\}$.

Proof. Since the block $b$ of $k G$ is not nilpotent, there exists a $b$-Brauer pair $\left(Q, e_{Q}\right)$ with $Q \neq 1$ such that $k N_{G}\left(Q, e_{Q}\right) e_{Q}$ is not nilpotent. This is because for some nontrivial Brauer pair $\left(Q, e_{Q}\right), N_{G}\left(Q, e_{Q}\right) / Q C_{G}(Q)$ is not a $p$-group. Amongst all such $b$-Brauer pairs, choose $\left(Q, e_{Q}\right)$ such that a defect group $R$ of $k N_{G}\left(Q, e_{Q}\right) e_{Q}$ has maximal order. After replacing, if necessary, $\left(Q, e_{Q}\right)$ by a suitable $G$-conjugate, we may assume that $R=N_{P}(Q)$. We are going to show that $R=P$, or equivalently that $P \subseteq N_{G}\left(Q, e_{Q}\right)$. We assume that $R$ is a proper subgroup of $P$, and derive 
a contradiction. Set $H=N_{G}\left(Q, e_{Q}\right)$. Clearly $R \subseteq H$. Since $Q \subset R$, we have $C_{G}(R) \subset C_{G}(Q) \subset H$. Now $\left(Q, e_{Q}\right) \subseteq\left(R, e_{R}\right)$, and $Q$ is normal in $R$, hence $e_{Q}$ is the unique block of $k C_{G}(Q)$ which is $R$-stable and for which $\operatorname{Br}_{R}\left(e_{Q}\right) e_{R}=e_{R}$ (cf. [1]).

Set $M=N_{G}(Z(J(R)))$. Since $C_{G}(R)$ centralises $Q$ and centralises $Z(J(R))$, we have $C_{G}(R) \subset M \cap H$. Let $d$ be the unique block of $k(M \cap H)$ (having $R$ as defect group) such that $\operatorname{Br}_{R}(d) e_{R}=e_{R}$. Let $f$ be the unique block of $k M$ (having $R$ as defect group) such that $\operatorname{Br}_{R}(f) e_{R}=e_{R}$. Since $Z(J(R))$ is a normal $p$-subgroup of $M, f$ is a central idempotent of $k C_{G}(Z(J(R)))$ (cf. [1]). Thus there exists a block $f_{0}$ of $C_{G}(Z(J(R)))$ such that $f f_{0}=f_{0}$ and $\left(Z(J(R)), f_{0}\right) \subseteq\left(R, e_{R}\right)$ in $M$, and hence in $G$. Since $\left(R, e_{R}\right) \subseteq\left(P, e_{P}\right)$, by the uniqueness of inclusion of Brauer pairs, we must have $f_{0}=e_{Z(J(R))}$. Let $M^{\prime}$ be the stabiliser of $e_{Z(J(R))}$ in $M$. Then $N_{P}(Z(J(R)))$, and hence $N_{P}(R)$ is contained in a defect group of $k M^{\prime} e_{Z(J(R))}$. In particular, the defect groups of $k M^{\prime} e_{Z(J(R))}$ have order strictly greater than $|R|$. By the maximality of $|R|$, we have that $k M^{\prime} e_{Z(J(R))}$ is nilpotent. Since $k M f$ is the induced algebra $\operatorname{Ind}_{M^{\prime}}^{M}\left(k M^{\prime} e_{Z(J(R))}\right)$, it follows that $k M f$ is nilpotent. Now $R C_{G}(Q) \subseteq M \cap H \subseteq N_{M}(Q)$, and by statement 1 again, it follows that $k(M \cap H) d$ is nilpotent. By the minimality of $|G|$, and the fact that $k H e_{Q}$ is not nilpotent, it follows that $H=G$ and hence $R=P$, contradicting the assumption $R \neq P$. If $R=P$, then $H$ satisfies the hypothesis of 2 with $d=e_{Q}$, and $k H e_{Q}$ is not nilpotent, thus $G=H$. In particular, $Q \subseteq O_{p}(G) \neq 1$.

From now on set $Q=O_{p}(G)$.

4. We have $G=N_{G}\left(Q, e_{Q}\right)$ and $b=e_{Q}$.

Proof. Since $G=N_{G}(Q)$, the block $b$ is contained in $k C_{G}(Q)$ (cf. [1]) and hence $b=$ $\operatorname{Tr}_{N_{G}\left(Q, e_{Q}\right)}^{G}\left(e_{Q}\right)$. Thus $k G b \cong \operatorname{Ind}_{N_{G}\left(Q, e_{Q}\right)}^{G}\left(k N_{G}\left(Q, e_{Q}\right) e_{Q}\right)$, so that in particular, $k N_{G}\left(Q, e_{Q}\right) e_{Q}$ is not nilpotent. Since $P$ is contained in $N_{G}\left(Q, e_{Q}\right)$, it follows from 2 that $G=N_{G}\left(Q, e_{Q}\right)$ and hence $b=e_{Q}$.

Note that $b$ is a block of any subgroup of $G$ containing $C_{G}(Q)$. We want to show that actually the pair $(Q, b)$ is $b$-centric (or self-centralising in the terminology of Puig, cf. [7, §41]); that is, the block $k C_{G}(Q) b$ is nilpotent with $Z(Q)$ as defect group. This notion goes back to Brauer [2]. We need the following technical statement.

5. Let $H$ be a subgroup of $G$ containing $P$ and let $d$ be a block of $k H$ having $P$ as defect group. Put $\bar{H}=H / Q$ and for any element a of $k H$ let $\bar{a}$ denote the image of a under the canonical surjection of $k H$ onto $k \bar{H}$. Then $\overline{\operatorname{Br}_{P}(d)}=\operatorname{Br}_{\bar{P}}(\bar{d})$.

Proof. Since $Q$ is normal in $H$, the block idempotent $d$ is a $k$-linear combination over the set $C_{H}(Q)_{p^{\prime}}$ of $p^{\prime}$-elements in $C_{H}(Q)$. Write $d=\sum_{g \in C_{H}(Q)_{p^{\prime}}} \alpha_{g} g$ with coefficients $\alpha_{g} \in k$. So $\bar{d}=\sum_{g \in C_{H}(Q)_{p^{\prime}}} \alpha_{g} \bar{g}$ and $\operatorname{Br}_{\bar{P}}(\bar{d})=\sum_{g \in C_{H}(Q)_{p^{\prime}} \cap C_{H}(\bar{P})} \alpha_{g} \bar{g}$, where $C_{H}(\bar{P})$ denotes the inverse image in $H$ of $C_{\bar{H}}(\bar{P})$.

We claim that $C_{H}(Q)_{p^{\prime}} \cap C_{H}(\bar{P})=C_{H}(P)_{p^{\prime}}$. To see this, consider the action of an element $g \in C_{H}(Q)_{p^{\prime}} \cap C_{H}(\bar{P})$ on an element $u$ of $P$. Since $g$ normalises $P$ and centralises $P / Q,{ }^{g}(u)=u v$ for some $v$ in $Q$. Let $n$ be the order of $g$. Since $g$ centralises $Q$, it follows that $u=g^{n} u=u v^{n}$. But $p$ and $n$ are relatively prime, hence $v=1$, thereby proving the claim.

The statement is immediate from the above expression for $\bar{d}$. 


\section{The blocks $k P C_{G}(Q) b$ and $k C_{G}(Q) b$ are nilpotent.}

Proof. By a result of Cabanes [4, normal $p$-extensions of nilpotent blocks are nilpotent; thus $k P C_{G}(Q) b$ is nilpotent if and only if $k C_{G}(Q) b$ is nilpotent. If $P C_{G}(Q)$ is a proper subgroup of $G$, then, by $2, b$ is nilpotent as a block of $P C_{G}(Q)$, and hence of $C_{G}(Q)$. Thus we may assume that $G=P C_{G}(Q)$. We have to show that $k G b$ is a nilpotent block. Set $\bar{G}=G / Q$ and let $\bar{b}$ denote the image of $b$ under the canonical surjection of $k G$ onto $k \bar{G}$. Identify $C_{G}(Q) / Z(Q)$ with its canonical image in $\bar{G}$; this is a normal subgroup of $\bar{G}$ of index a $p$-power. Since $b$ is a $k$-linear combination of $p^{\prime}$-elements in $C_{G}(Q)$ and $Z(Q)=Q \cap C_{G}(Q)$ is a central subgroup of $C_{G}(Q)$, it is clear that $\bar{b}$ is a block of $k C_{G}(Q) / Z(Q)$ and hence of $k \bar{G}$. Furthermore, $\bar{P}$ is a defect group of $k \bar{G} \bar{b}$. Let $Z$ be the inverse image in $G$ of $Z(J(\bar{P}))$ and set $H=N_{G}(Z)$. Then $H$ is the inverse image in $G$ of the group $\bar{H}=k N_{\bar{G}}(Z(J(\bar{P})))$. Let $f$ be the block of $k \bar{H}$ which corresponds to the block $\bar{b}$ of $k \bar{G}$; that is, $\operatorname{Br}_{\bar{P}}(\bar{b})=\operatorname{Br}_{\bar{P}}(f)$. Clearly, $P$ and $C_{G}(Z)$ are both subgroups of $H$. Since $Z$ properly contains $Q$ and $Q=O_{p}(G), H$ is a proper subgroup of $G$. Thus by 2 , the block $k H d$ is nilpotent where $d$ is the block of $k H$ satisfying $\operatorname{Br}_{P}(d) e_{P}=e_{P}$. Since $N_{G}(P)$ is contained in $H$, we have in fact that $\operatorname{Br}_{P}(d)=\operatorname{Br}_{P}(b)$.

Now, it follows from 5 that

$$
\operatorname{Br}_{\bar{P}}(\bar{d})=\overline{\operatorname{Br}_{P}(d)}=\overline{\operatorname{Br}_{P}(b)}=\operatorname{Br}_{\bar{P}}(\bar{b})=\operatorname{Br}_{\bar{P}}(f) .
$$

In particular, $\bar{d} f \neq 0$. Since $k H d$ is nilpotent, this means that $f=\bar{d}$ and hence that $k \bar{H} f$ is nilpotent. As $G$ is a minimal counterexample to the Theorem, it follows that $k \bar{G} \bar{b}$ is nilpotent, which implies that $k G b$ is nilpotent.

7. The group $Q$ is a defect group of $k Q C_{G}(Q) b$.

Proof. Let $R$ be a defect group of $k Q C_{G}(Q) b$. We may assume that $R=Q C_{P}(Q)$. The pair $\left(R, e_{R}\right)$ is a maximal Brauer pair for the block $k Q C_{G}(Q) b$, and hence, by the Frattini argument,

$$
G=N_{G}\left(R, e_{R}\right) Q C_{G}(Q)=N_{G}\left(R, e_{R}\right) C_{G}(Q) .
$$

Suppose, if possible, that $Q$ is a proper subgroup of $R$. Then, $N_{G}\left(R, e_{R}\right)$ is a proper subgroup of $G$ because $Q=O_{p}(G)$. On the other hand, $N_{G}\left(R, e_{R}\right)$ satisfies the hypothesis of 2 with $R$ instead of $Q$, since $P$ normalises $R$, and consequently $\left(R, e_{R}\right)$. So $k N_{G}\left(R, e_{R}\right) e_{R}$ is nilpotent. In particular, $N_{G}\left(R, e_{R}\right) / C_{G}(R)$ is a $p$ group, and hence so is $G / C_{G}(Q)$. In other words, $G=P C_{G}(Q)$, and hence $k G b$ is nilpotent by 6 , a contradiction.

We are now in the situation where $k G b$ is an extension of the nilpotent block $k Q C_{G}(Q) b$, and this is where the results of Külshammer and Puig in [6] come in.

8. There exists a short exact sequence of groups

$$
1 \longrightarrow Q \longrightarrow L \longrightarrow G / Q C_{G}(Q) \longrightarrow 1
$$

such that $P$ is a Sylow p-subgroup of $L$ and such that we have $\mathcal{F}_{G, b}=\mathcal{F}_{L}$.

Proof. Note first that $P$ is also a defect group of $\{b\}$ viewed as a point of $G$ on $\mathcal{O} Q C_{G}(Q)$ because $P$ is maximal with the property $\operatorname{Br}_{P}(b) \neq 0$. The existence of a canonical short exact sequence of finite groups as stated such that $P$ is a Sylow- $p$ - 
subgroup of $L$ is a particular case of [6, 1.8]. The equality $\mathcal{F}_{G, b}=\mathcal{F}_{L}$ is a translation of the statement [6, 1.8.2], which requires a brief explanation. Since $Q$ is normal in $L$ and in $G$, it suffices to show that the images in $\operatorname{Aut}(R)$ of $N_{G}\left(R, e_{R}\right) / C_{G}(R)$ and $N_{L}(R) / C_{L}(R)$ are equal, where $R$ is a subgroup of $P$ containing $Q$. As $\left(Q, e_{Q}\right)$ is $b$ centric and $Q$ is $p$-centric in $L$, it follows from a result of Puig (cf. [7, (41.1), (41.4)]) that $\left(R, e_{R}\right)$ is $b$-centric and $R$ is $p$-centric in $L$ (that is, $Z(R)$ is a Sylow-p-subgroup of $C_{L}(R)$ ). In particular, $k C_{G}(R) e_{R}$ has a unique conjugacy class of primitive idempotents. Setting $H=Q C_{G}(Q)$, we have $C_{G}(R)=C_{H}(R)$, hence there is a unique point $\gamma_{R}$ of $R$ on $k H$ such that $\operatorname{Br}_{R}(i) e_{R}=i$ for some (and hence any) element $i$ of $\gamma_{R}$. In this way, we get an inclusion preseving bijection, $R_{\gamma_{R}} \rightarrow\left(R, e_{R}\right)$ between local pointed groups $R_{\gamma_{R}}$ on $k H b$ for which $Q_{\gamma_{Q}} \subseteq R_{\gamma_{R}} \subseteq P_{\gamma_{P}}$ and $k G b$ Brauer pairs, $\left(R, e_{R}\right)$ with $\left(Q, e_{Q}\right) \subseteq\left(R, e_{R}\right) \subseteq\left(P, e_{P}\right)$. Further, it is clear that $N_{G}\left(R, e_{R}\right)=N_{G}\left(R_{\gamma_{R}}\right)$. Thus, setting $\bar{G}=G / Q C_{G}(Q)$, with the notation in [6, 1.8] (which is defined in [6, 2.8]), we have $E_{G, \bar{G}}\left(R, e_{R}\right)=E_{L, \bar{G}}(R)$ for any subgroup $R$ such that $Q \leq R \leq P$. By [6. (2.8.1)], the canonical maps $E_{G, \bar{G}}\left(R, e_{R}\right) \rightarrow$ $E_{G}\left(R, e_{R}\right)$ and $E_{L, \bar{G}}(R) \rightarrow E_{L}(R)$ are surjective. Thus $E_{G}\left(R, e_{R}\right)=E_{L}(R)$, which implies the equality $\mathcal{F}_{G, b}=\mathcal{F}_{L}$.

9. We have $\mathcal{F}_{N, c}=\mathcal{F}_{N_{L}(Z(J(P)))}$.

Proof. Since $Z(J(P))$ is normal in both $N$ and $N_{L}(Z(J(P)))$, it suffices to show that the images of $N_{G}(S, f) \cap N$ and $N_{L}(S) \cap N_{L}(Z(J(P)))$ in Aut $(S)$ are equal, where $(S, f)$ is a $c$-Brauer pair contained in $\left(P, e_{P}\right)$ such that $Z(J(P)) \subseteq S$. Note that then $C_{G}(S) \subseteq N$ and hence $f=e_{S}$. Also, by 8 we have $\mathcal{F}_{G, b}=\mathcal{F}_{L}$. Thus for any $x \in N_{G}\left(S, e_{S}\right)$ there is $y \in N_{L}(S)$ such that ${ }^{x} u={ }^{y} u$ for all $u \in S$. Since $Z(J(P)) \subseteq S$ we have $x \in N_{G}\left(S, e_{S}\right) \cap N$ if and only if $y \in N_{L}(S) \cap N_{L}(Z(J(P)))$, from which the equality 9 follows.

We conclude the proof of the Theorem as follows. Since $k G b$ is not nilpotent, $L$ is not a $p$-nilpotent group by 8 . However, $k N c$ is nilpotent and hence $N_{L}(Z(J(P)))$ is $p$-nilpotent by 9 . This contradicts the normal $p$-complement theorem [5, Ch. 8 , Theorem 3.1] of Glauberman and Thompson.

\section{ACKNOWLEDGEMENTS}

This work was done while the second author was a visitor at the Mathematical Institute of the University of Oxford and he would like to thank the institute for its hospitality.

\section{REFERENCES}

1. J. L. Alperin, M. Broué, Local methods in block theory, Ann. Math. 110 (1979), 143-157. MR 80f:20010

2. R. Brauer, On the structure of blocks of characters of finite groups, Lecture Notes in Mathematics 372 (1974), 103-130. MR 50:4725

3. M. Broué, L. Puig, A Frobenius theorem for blocks, Invent. Math. 56 (1980), 117-128. MR 81d:20011

4. M. Cabanes, Extensions of p-groups and construction of characters, Comm. Alg. 15 (1987), 1297-1311. MR 88b:20023

5. D. Gorenstein, Finite Groups, Second edition, Chelsea Publishing Company, New York, 1980. MR 81b:20002 
6. B. Külshammer, L. Puig, Extensions of nilpotent blocks, Invent. Math. 102 (1990), 17-71. MR 91i:20009

7. J. Thévenaz, G-Algebras and Modular Representation Theory, Oxford Science Publications, Clarendon Press, Oxford, 1995. MR 96j:20017

Department of Mathematics, University College, High Street, Oxford OX14BH, United Kingdom

Current address: Department of Mathematics, The Ohio State University, 231 W. 18th Avenue, Columbus, Ohio 43210

CNRS, Université Paris 7, UfR Mathématiques, 2, place Jussieu, 75251 Paris Cedex 05, FRANCE

Current address: Department of Mathematics, The Ohio State University, 231 W. 18th Avenue, Columbus, Ohio 43210 